\title{
Introducing the Interdisciplinary Nature of Health Care Through Case Study Models
}

\author{
Kathryn T. Newton ${ }^{1}$, Aaron Ashley ${ }^{2}$ \\ ${ }^{1}$ Department of Health Science, Weber State University, Ogden, Utah, USA \\ ${ }^{2}$ Department of Psychology, Weber State University, Ogden, Utah, USA \\ Correspondence: Kathryn T. Newton, Department of Health Science, Weber State University, Ogden, Utah 84408-3909, \\ USA.
}

Received: November 3, 2019

Accepted: December 3, 2019 Online Published: December 5, 2019

doi:10.11114/jets.v8i1.4600

URL: https://doi.org/10.11114/jets.v8i1.4600

\begin{abstract}
Interprofessional education (IPE) is a critical area needed to improve the quality of healthcare. Stereotypes of other disciplines persist by healthcare workers, limiting the ability to work as a team. Knowledge of roles of healthcare professions is a key competency of IPE. An online, 12-week course was developed to introduce students to the interdisciplinary nature of healthcare through case study models. Through built-in links, students explore medical terminology, diagnostic testing, pathophysiology, treatment, and health professionals who work as a team to diagnose and treat the patient. A 24-question survey was administered to a convenience sample of 582 high school students. Results of paired-samples t-tests showed significant increases pre-training to post-training in: 1) knowledge of the different roles of health professionals, $(n=338, M=.71$, SEM $=.01)$ to post-training $(M=.82$, SEM $=.01), t(337)=$ $\left.11.08, p<.001, p \eta^{2}=.203,2\right)$ and self-reported knowledge of roles of different health professions, pre-training $(n=338$, $M=2.73, S E M=.30)$ to post-training $(M=3.60, S E M=.039), t(337)=20.02, p<.001, p \eta^{2}=.543$. Interestingly, students' likelihood in a career in healthcare professions decreased significantly pre-training $(n=338, M=4.30, S E M$ $=.043)$ to post-training $(M=4.20, S E M=.06), t(337)=2.21, p=.028, p \eta^{2}=.016$. Introducing knowledge of health professions to this population may be critical in avoiding stereotypes before a student enters a chosen career path, increasing the likelihood of participation in interdisciplinary teams.
\end{abstract}

Keywords: interprofessional education, allied health professions, case-based learning, concurrent enrollment, course design, online education

\section{Introduction}

At the turn of the $21^{\text {st }}$ century, The Institute of Medicine [IOM] (2001) called for major reforms in the American health care system advocating for changes in the quality of care and effective use of resources. "Between the health care we have and the care we could have lies not just a gap, but a chasm" (IOM, 2001). One of the factors leading to this gap in care is the changing nature of the patient. With scientific and technological advancements, people are living longer. When the 2001 IOM report was released, about 1 in 8 persons, or $13 \%$ of the population in the United States, were over 65 years of age. In 2018, that number rose to $16 \%$ of the population and projections are that it will rise to $23 \%$ by 2060 (Population Reference Bureau, 2019). With people living longer, the shift in leading causes of disease are no longer acute, but chronic. These individuals often present with comorbid conditions, complicating their healthcare. The percentage of individuals over 65 reporting comorbid conditions increased from $20.2 \%$ in 2005 to $28 \%$ in 2014 . The most common health conditions include cardiovascular disease, diabetes, cancer, and chronic obstructive pulmonary diseases (Atella et al., 2019). This population has a corresponding higher utilization of healthcare, higher expenditures, with poorer health outcomes (Palladino, Lee, Ashworth, Triassi, \& Millett, 2016).

Addressing gaps in healthcare with a changing population is challenging. Rather than simply finding a cure, treating chronic disease involves controlling symptoms while managing chronic care, and maximizing quality of life (Hall \& Weaver, 2001). The IOM (2001) called for healthcare professionals to work together in teams. In the last century, a family physician often was responsible for all of an individual's healthcare needs. With changing demographics, a team approach is more effective and may include different physicians, nurses, social workers, diagnostic technicians, and others (Nester, 2016). The Mayo Clinic is an example of a nonprofit hospital that promotes team-based medicine. 
"Patients who come to Mayo Clinic with complex medical conditions benefit from the pooling of knowledge within the organization that is relevant to their care - they get the benefit of an integrated team" (Berry \& Beckham, 2014). Mitchell et al., (2019) reported findings on Mayo Clinic's implementation of a primary care clinic that utilized team-based care. Teams are comprised of five physicians, ten advanced practice practitioners, registered nurses, behavioral health providers, and community specialists. In a two-year study, patient satisfaction increased from $82.6 \%$ to $89.4 \%$. Patient costs slightly decreased and team satisfaction had a mean positive response of $80 \%$. A study of patients with multiple chronic health conditions in a federally funded health center demonstrated a $70 \%$ patient satisfaction when treated with a multi-disciplinary healthcare team (Smith, Johnson-Lawrence, Andrews, \& Parker, 2017).

With the growing need and success of interdisciplinary healthcare teams, it is imperative that education also shifts to emphasize interdisciplinary teams. The need for interprofessional education (IPE) was highlighted as a critical need to improve healthcare in America (IOM, 2001). IPE has been defined as "two or more professions who learn with, from and about each other to improve collaboration and quality of care" (Barr, 2002).

Limited knowledge and stereotypes of others in the healthcare team persist, especially regarding clinical knowledge and ability. Hean, Clark, Adams, \& Humphris (2006) found that students entering their first year of university studies had formed distinct stereotypical attitudes of health professional groups, and subsets of these groups. Midwives, social workers and nurses were ranked highest on interpersonal skills and on being team players. Physicians and pharmacists ranked highest on academic ability and lowest on being team players. Doctors were also given high scores on leadership ability. Interestingly, nurses were ranked lower on their ability to make decisions. Michalec, Giordano, Arenson, Antony, \& Rose, (2013), found that inaugural students in individual health professions rated their own discipline highest in almost every measureable attribute, causing them to view every other discipline as less favorable, making it difficult to work as a healthcare team. Ekmekci (2013) found when IPE components were incorporated into a student's curriculum; the tendency for stereotyping decreased significantly compared to students without the IPE components.

Knowledge of the roles of various health professions has been identified as a key competency of IPE (MacDonald et al., 2010; Bryne \& Pettigrew, 2010). This competency can be broken down to indicators of behavior:

- The individual can "describe where the scope of one's own profession ends and another begins"

- Is open to and seeks out contributions of other team members

- Addresses misconceptions among team members

- Respects the roles, expertise, and contributions of others in the team

- Identifies overlapping professional skills

- Values the collaborative efforts of the team, and

- Can describe the different perspectives and knowledge of other professions" (MacDonald et al., 2010).

It stands to reason that if knowledge can be introduced before a student begins pursuing their individual discipline, they may be less likely to form negative stereotypes and more likely to welcome a team approach to medicine.

A number of programs have demonstrated the value of introducing high school students to a variety of health professions. Afghani, Santos, Angulo, \& Muratori (2013) utilized faculty and medical students to mentor high school students in a summer program, increasing interest of the students in pursuing a health profession. Zavattieri, D'Anna, \& Maillet (2007) looked at the longitudinal effects of a high school Health Science careers program, finding that approximately half of the participants pursued a career in health professions. Other programs have focused on introducing minorities to healthcare fields (Gefter et. al., 2018; Balogun, Sloan, \& Hardney, 2005; Derck, Zahn, Finks, Mand, \& Sandhu, 2016; Danner, et. al, 2017).

A natural conclusion would be that students' knowledge of the roles and responsibilities of various health professions would increase in these outreach programs, yet the literature shows little reporting in changes in knowledge. In addition, the majority of studies did not focus on interventions that introduced the interprofessional nature of healthcare to the high school population. One exception is a study by Hayward, Canali, \& Hill (2005) which utilized high school seniors, paired with health profession students, to develop interdisciplinary case studies. A faculty member working with the groups commented that it was beneficial for the students to learn how "different medical professions work together and who is responsible for what part of the patient's care." Lindqvist, Duncan, Shepstone, Watts, \& Pearce (2005) also found significant changes in attitude towards other health professions, when interdisciplinary teams worked through case studies.

Case Based Learning (CBL) may be an effective means to introduce health professionals as a team to the high school population. CBL is an effective means to impart relevance, and aid in connecting theory to practice. Dowd \& 
Davidhizar (1999) argue that CBL promotes critical thinking. By giving students experience in solving case scenarios, students accumulate knowledge that is more applicable in the clinical setting. In a systematic review of CBL in health professional education, students overwhelmingly felt this methodology enhanced their learning (Thistlethwaite et. al, 2012). Impact can range from simple knowledge gains to changing patient practice (McLean, 2016). It is reasonable to conclude that students may increase knowledge of the different roles of health professionals through case scenarios built on evidence-based medicine.

Through our literature review, we have not found an intervention that introduces secondary students to the roles of members of the healthcare team through case-based learning. In response to these findings, we developed a unique online course that introduces the interdisciplinary roles of health professionals through case studies. The purpose of this study is to 1) assess if students' knowledge of the responsibilities of different health professions changes from pre- to post-training, 2) assess if self-reported knowledge of different health professions changes from pre- to post-training, 3) assess if students' interest in pursuing a health profession changes over the course of study.

\section{Material and Method}

\subsection{Subjects}

An online course introducing students to health professionals through medical case studies was offered to secondary students as concurrent enrollment (CE) at 25 high schools in Utah, and also offered as an elective course for pre-program Allied Health university students. CE courses are defined as those in which a student may gain both high school and university credit. The course is typically offered as part of a block of Health Science courses, designated by the Utah State Board of Education as "Career and Technical Education (CTE) pathways (Utah State Board of Education, 2019). Course offerings include biotechnology, medical anatomy and physiology, medical math, nutrition, and medical terminology.

Five hundred and eighty two subjects enrolled in the study. Subjects were limited to students enrolled in the Concurrent Enrollment course in the state of Utah. Participants were between 16-18 years of age. Seventy-nine percent of participants self-reported as Caucasian, 12\% Hispanic, 4\% Asian, 2\% Black or African American, 2\% Pacific Islander, and $1 \%$ mixed raced. These demographics are representative of Utah demographics reported in 2019 (Utah Population, 2019).

\subsection{Sampling Procedures}

High school instructors were given written instructions regarding the study, and asked to give recruitment flyers and consent forms to students participating in the course. Students were awarded 10 points of extra credit upon completion of the pre-training survey, and 10 points upon completion of the post-training survey. This represents $3 \%$ of the total points possible for the course. Students that opted out of the study were given the option of writing a one-page paper on a health profession of their choice for each survey, providing the opportunity of earning the same amount of extra credit. The Weber State University Institutional Review Board approved this study.

\subsection{Sample Size}

In order to achieve a 95\% confidence level and a 5\% margin of error, 377 participants were needed for the study. Researchers planned to recruit 600 subjects to account for attrition. Five hundred and eighty two subjects consented to the study and completed pre-training surveys. Post-training surveys were completed by 411 students, which represents a $29 \%$ attrition rate. Students from one of the participating high schools did not complete post-training surveys ( $\mathrm{n}=22$ ). There is also a typical $10-20 \%$ attrition rate from CE classes as students self-select out of the course during the semester.

\subsection{Measures}

A 24-question survey was developed to assess knowledge and interest of individual health professions introduced throughout the course (See Appendix). The survey was broken into three sections:

1. Thirteen questions assessing knowledge of the responsibilities of various health professions. These questions are scenario-based, multiple choice questions.

2. Ten Likert-scale questions providing a self-assessment of knowledge of individual health professions.

3. One question assessing interest in pursuing a health profession.

Pre- post-surveys were delivered through Qualtrics online surveys. Qualtrics protects data by securing their servers with firewalls and regularly performs scans to detect any vulnerabilities in their systems. High school instructors were trained in administering the surveys in a secure testing environment. Individual surveys were not identified by name. Only researchers involved in the study had access to the data. 


\subsection{Research Design}

This study utilized a within-subjects repeated measure design. Subjects completed a pre-training survey followed by a 12-week course (see course design, section 2.34). At the conclusion of the course, subjects completed the post-training survey. High school instructors are trained with course content and delivery individually at their time of appointment, and yearly at an annual CE instructor meeting. At this annual meeting, high school instructors were trained regarding recruitment of subjects, consent, and course delivery.

\subsection{Intervention/Course Design}

An online course was designed that introduces twelve medical case studies, focusing on patient case histories. Each case is presented as a patient story. Within the story, students review the 1) anatomy and physiology of the body system as it pertains to the case, 2) medical terminology, 3) patient symptoms 4) diagnostic testing, 5) the pathophysiology of the disease process, 6) treatment and 7) the health professionals who work as a team to diagnose and treat disease.

Table 1. Summary of Case Studies and Representative Body Systems

\begin{tabular}{ll}
\hline Case Study & Body System \\
Burn & Integumentary \\
Anterior Cruciate Ligament Tear & Muscular/Skeletal \\
Multiple Sclerosis & Nervous I \\
Meningitis & Nervous II and Infectious Disease \\
Type I Diabetes & Endocrine \\
Acute Lymphocytic Leukemia & Blood \\
Myocardial Infarction & Cardiovascular \\
Hemophilia & Blood/Genetics \\
Asthma & Respiratory \\
Hepatitis A and B & Gastrointestinal \\
Acute Renal Failure & Renal \\
Testicular Cancer & Reproductive \\
\hline
\end{tabular}

The case study topics with their accompanying body systems can be viewed in Table 1 . Within each case, there are links to supporting pages and video, allowing students to utilize medical sites on the web as well as supporting pages built for the course. This supporting material includes a built-in medical dictionary, overview of diagnostic testing methods, the disease process, and descriptions of each health profession, which explore the job description, educational requirements, compensation ranges, and future opportunities for each of the health professions presented. See Figure 1.

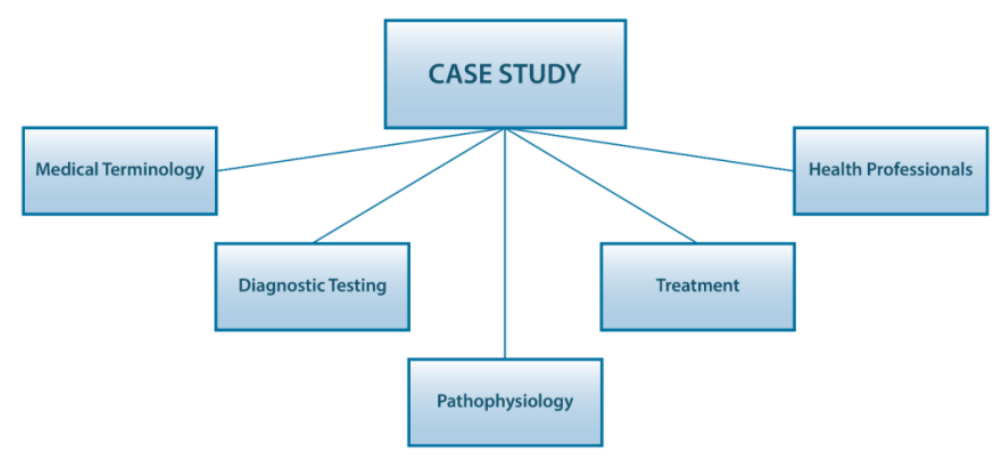

Figure 1. Case Study and Supporting Pages

Throughout each case study, the health professionals are presented as working together as a team. Figure 2 is an example of the health professionals interacting in a case study of a patient with a severe burn. 


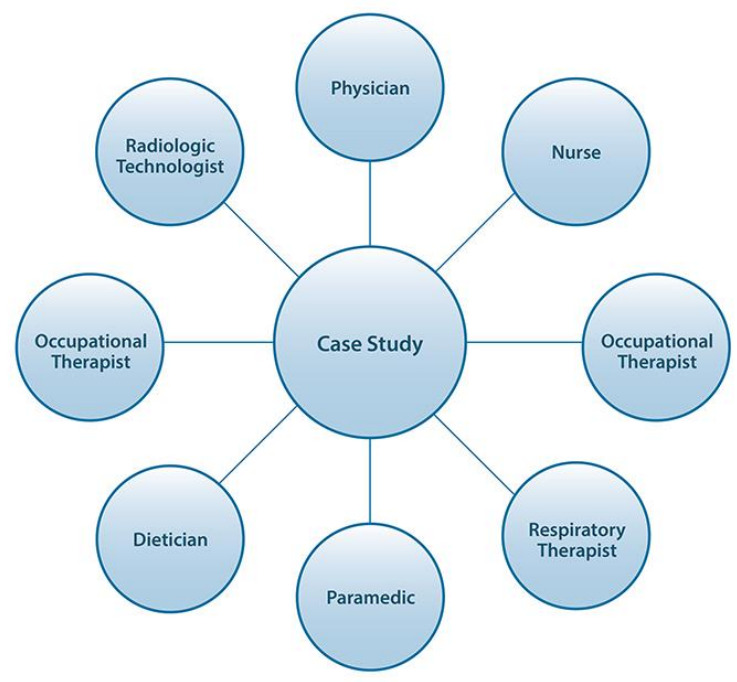

Figure 2. Interdisciplinary Health Care Team

Each case study concludes with a summary page. As part of the summary, the roles and responsibilities of each health professional in the case are delineated. As students explore the patient's story, they can observe the interactions of the health professionals involved in diagnosing and treating the patient.

Course assessment includes completion of study materials included in a workbook that accompanies the course. Students are also required to complete a pre-test and exam, which accompany each module.

This course was completed between 12-16 weeks of study, depending on the participating high school. Individual school districts vary in Utah with some being trimester based, and others semester based. High school delivery also varies with course offerings. Several high school districts offer the course in a composite two class period course, which includes Introductory Medical Anatomy and Physiology, CE Medical Terminology, and the CE Case Studies course described in this article. Other districts offer the course as a stand-alone one period course. The different delivery methods of this course were not controlled for in the study.

\section{Results}

The current study investigated the effect of training on 1) students' knowledge of the different roles of health care professionals, 2) students' self-reported knowledge of the responsibilities of different members of a healthcare team, and 3) students' interest in pursuing a career in the healthcare professions. These variables were computed from students' responses on a questionnaire (See Appendix). Students' knowledge of different roles was computed from percentage of correct responses on 13 multiple-choice questions (.25 probability correct response) about types of jobs conducted by different healthcare professions. Students' knowledge of healthcare team member responsibilities was computed from an average of responses to 10 questions on a 5 pt-scale $(1=$ "No Knowledge" to $5=$ "I know a lot about this profession"). Likelihood of pursuing a career in the healthcare profession was based on a single 5pt-scale question $(1=$ "Not likely" to $5=$ "Definitely") assessing likelihood of pursuing a healthcare profession post-high school.

Results of three paired-samples t-tests showed significant changes in all three measures from pre-training to post-training. The percentage of correct responses increased significantly from pre-training $(n=338, M=.71, S E M$ $=.01)$ to post-training $(M=.82, S E M=.01), t(337)=11.08, p<.001, p \eta^{2}=.203$. Additionally, students' self-reported knowledge of the role of professionals on a healthcare team increased from pre-training $(n=338, M=2.73, S E M=.30)$ to post-training $(M=3.60, S E M=.039), t(337)=20.02, p<.001, p \eta^{2}=.543$. Interestingly, students' likelihood in a career in healthcare professions decreased significantly pre-training $(n=338, M=4.30, S E M=.043)$ to post-training $(M=4.20, S E M=.06), t(337)=2.21, p=.028, p \eta^{2}=.016($ See Table 2$)$. 
Table 2. Student results pre- to post-test

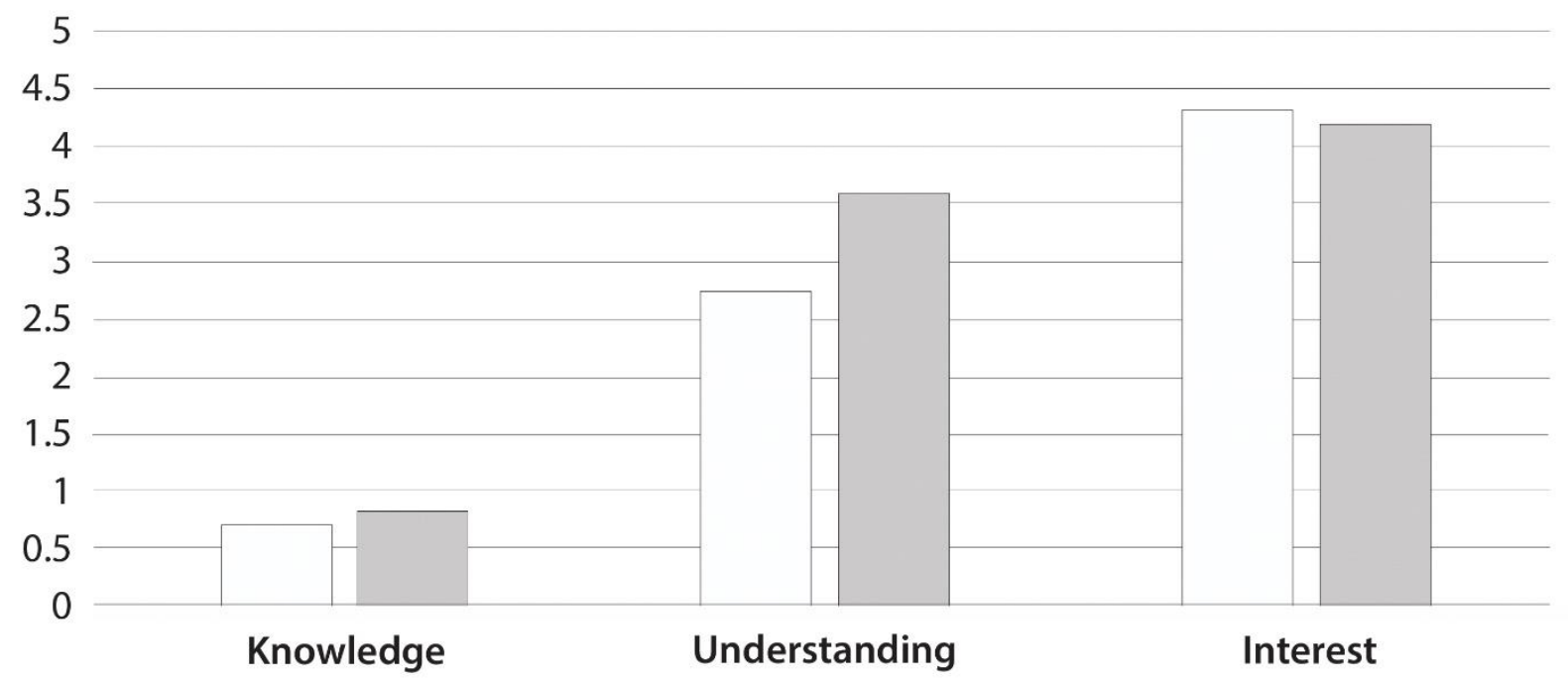

\section{Pre Training $\square$ Post Training}

\section{Discussion}

This study demonstrated significant effects on knowledge of the different roles of health professions and self-reported knowledge of the responsibilities of health professionals following the conclusion of the course. Increasing knowledge before a student enters their chosen discipline is important in decreasing negative stereotypes and increasing an open mind towards interdisciplinary teamwork. This course introduces a unique opportunity to introduce this knowledge early to secondary students, who are still exploring and forming opinions about their future chosen discipline. Interesting findings were that students' interest in choosing a healthcare profession decreased significantly after participating in the course. This finding has several possible explanations. Secondary students are just beginning to explore possible career paths. Gore et al., (2018) looked at variables that affect students' choice of a career in medicine with one of the variables being self-perception of academic performance. The course described in this article is a university level course, offered concurrently to secondary students. By nature, this course is difficult for this population. Less desirable academic performance in the Case Study course, as well as additional health career courses taken as part of the CTE pathway may influence a student's decision to change career paths from a health career. Ultimately, this may be to the student's benefit. Health Science career paths are rigorous, and may not be the best fit for a particular student. Figuring this out early, before investing time and money in college, may prove beneficial to the individual student.

Limitations of this study include the use of a convenience sample specific to this course and the state of Utah. The study was limited to students that speak English, and students that self-selected a career course cluster in Health Science. This study was limited to the assessment of knowledge of health profession roles and self-assessment of the knowledge of those roles. The tool used for the survey was developed based on information presented in the course, limiting its applicability to other interventions. Confounding variables that were not controlled for include time spent on the course, and other courses taken concurrently. Both may have had an effect on individual outcomes.

Future studies should assess other dependent variables including attitudes and knowledge of the interdisciplinary roles of health professionals. Of interest would also be an assessment of the impact of the course on knowledge and interest in pursuing "silent" health professions, professions that may not be as well known, but are still an important part of the team. A longitudinal study following a cohort of students that complete this course would be of interest, specifically, is there an impact of the course on a student's chosen profession and knowledge and attitudes towards others in the healthcare team. Future studies should also assess differences in knowledge of health profession roles, and attitudes towards different health professions, between the high school population, and pre-program allied health students.

Researchers should continue to look for ways to introduce secondary students to the interdisciplinary nature of the healthcare team. By introducing this knowledge early, it could be hypothesized that students' stereotyping of other health professions would decrease, thus increasing positive attitudes towards working as a healthcare team. 


\section{References}

Afghani, B., Santos, R., Angulo, M., \& Muratori, W. (2013). A novel enrichment program using cascading mentorship to increase diversity in the health care professions. Academic Medicine: Journal of the Association of American Medical Colleges, 88(9), 1232. https://doi.org/10.1097/ACM.0b013e31829ed47e

Atella, V., Piano Mortari, A., Kopinska, J., Belotti, F., Lapi, F., Cricelli, C., \& Fontana, L. (2019). Trends in age-related disease burden and healthcare utilization. Aging cell, 18(1), e12861. https://doi.org/10.1111/acel.12861

Balogun, J. A., Sloan, P. E., \& Hardney, K. (2005). Health professions career awareness program for seventh-and eighth-grade african-american students: A pilot study. Journal of Allied Health, 34(4), 236-243.

Barr, H. (2002). Interprofessional education: Today, yesterday and tomorrow. Occasional Paper No. 5 London: Centre for the Advancement of Interprofessional Education.

Berry, L. L, \& Beckham, D. (2014). Team-based care at mayo clinic: A model for ACOs. Journal of Healthcare Management, 59(1), 9-13. https://doi.org/10.1097/00115514-201401000-00003

Byrne, Á., \& Pettigrew, C. M. (2010). Knowledge and attitudes of allied health professional students regarding the stroke rehabilitation team and the role of the Speech and Language Therapist. International Journal of Language \& Communication Disorders, 45(4), 510-521. https://doi.org/10.3109/13682820903222791

Danner, O. K., Lokko, C., Mobley, F., Dansby, M., Maze, M., Bradley, B., ... Childs, E. (2017). Hospital-based, Multidisciplinary, youth mentoring and medical exposure program positively influences and reinforces health care career choice: "The Reach One Each One Program early Experience." American Journal of Surgery, 213(4), 611-616. https://doi.org/10.1016/j.amjsurg.2016.12.002

Derck, J., Zahn, K., Finks, J. F., Mand, S., \& Sandhu, G. (2016). Doctors of tomorrow: An innovative curriculum connecting underrepresented minority high school students to medical school. Education for Health (Abingdon, England), 29(3), 259-265. https://doi-org.hal.weber.edu/10.4103/1357-6283.204219

Dowd, S. B., \& Davidhizar, R. (1999). Using Case Studies to Teach Clinical Problem-Solving. Nurse Educator, 24(5), 42-46. https://doi.org/10.1097/00006223-199909000-00017

Ekmekci, O. (2013). Promoting Collaboration in Health Care Teams through Interprofessional Education: A Simulation Case Study. International Journal of Higher Education, 2(1), 78-83. https://doi.org/10.5430/ijhe.v2n1p78

Gefter, L., Spahr, J., Gruber, J., Ross, S., Watson, L., \& Mann, B. (2018). Addressing health disparities with schoolbased outreach: The health career academy program. Journal of Racial and Ethnic Health Disparities, 5(4), 700-711. https://doi.org/10.1097/00006223-199909000-00017

Gore, J., Patfield, S., Holmes, K., \& Smith, M. (2018). Widening participation in medicine? New insights from school students' aspirations. Medical Education, 52(2), 227-238. https://doi.org/10.1111/medu.13480

Hall, P., \& Weaver, L. (2001). Interdisciplinary education and teamwork: a long and winding road. Medical Education, 35(9), 867-875. https://doi.org/10.1046/j.1365-2923.2001.00919.x

Hayward, L. M., Canali, A., \& Hill, A. (2005). Interdisciplinary peer mentoring: A model for developing culturally competent health care professionals. Journal of Physical Therapy Education,19(1), 28-40. https://doi.org/10.1097/00001416-200501000-00005

Hean, S., Clark, J. M., Adams, K., \& Humphris, D. (2006). Will opposites attract? Similarities and differences in students' perceptions of the stereotype profiles of other health and social care professional groups. Journal of Interprofessional Care, 20(2), 162-181. https://doi.org/10.1080/13561820600646546

Institute of Medicine. Committee on Quality of Health Care in America. (2001). Crossing the quality chasm: a new health system for the 21st century. Washington, DC: The National Academies Press.

Lindqvist, S., Duncan, A., Shepstone, L., Watts, F., \& Pearce, S. (2005). Case-based learning in cross-professional groups - the development of a pre-registration interprofessional learning programme. Journal of Interprofessional Care, 19(5), 509-520. https://doi.org/10.1080/13561820500126854

MacDonald, M. B., Bally J. M., Ferguson, L. M., Murray, B. L, Fowler-Kerry, S. E., \& Anonson, J. M. (2010). Knowledge of the professional role of others: A key interprofessional competency. Nurse Education in Practice, 10(4), 238-242. https://doi.org/10.1016/j.nepr.2009.11.012

McLean, S. F. (2016). Case-Based Learning and its Application in Medical and Health-Care Fields: A Review of Worldwide Literature. Journal of Medical Education \& Curricular Development, (3), 39-49. https://doi.org/10.4137/JMECD.S20377 
Michalec, B., Giordano, C., Arenson, C., Antony, R., \& Rose, M. (2013). Dissecting first-year students' perceptions of health profession groups: potential barriers to interprofessional education. Journal of Allied Health, 42(4), 202-213.

Mitchell, J. D., Haag, J. D., Klavetter, E., Beldo, R., Shah, N. D., Baumbach, L. J., ... Stroebel, R. J. (2019). Development and implementation of a team-based, primary care delivery model: Challenges and opportunities. Mayo Clinic Proceedings, 94(7), 1298-1303. https://doi.org/10.1016/j.mayocp.2019.01.038

Nester, J. (2016). The importance of interprofessional practice and education in the era of accountable care. North Carolina Medical Journal, 77(2), 128-132. https://doi.org/10.18043/ncm.77.2.128

Palladino, R., Tayu Lee, J., Ashworth, M., Triassi, M., \& Millett, C. (2016). Associations between multimorbidity, healthcare utilization and health status: evidence from 16 European countries. Age and Ageing, 45(3), 431-435. https://doi.org/10.1093/ageing/afw044

Population Reference Bureau (2019). Fact Sheet: Aging in the United States. Retrieved November 30, 2019, from https://www.prb.org/aging-unitedstates-fact-sheet/

Smith, L. J., Johnson-Lawrence, V., Andrews, M., \& Parker, S. (2017). Opportunity for interprofessional collaborative care-findings from a sample of federally qualified health center patients in the midwest. Public Health, 151, 131-136. https://doi.org/10.1016/j.puhe.2017.07.009

Thistlethwaite, J. E., Davies, D., Ekeocha, S., Kidd, J. M., MacDougall, C., Matthews, P., ... Clay, D. (2012). The effectiveness of case-based learning in health professional education. A BEME systematic review: BEME Guide No. 23. Medical Teacher, 34(6), e421-e444. https://doi.org/10.3109/0142159X.2012.680939

Utah Population (2019, October 29). In World Population Review. Retrieved from October 29, 2019 , https://doi.org/10.1144/geosci-29-9

Utah State Board of Education (2019, November 2). In Utah Career and Technical Education Career Pathway. Retrieved November 2, 2019, from https://www.schools.utah.gov/file/ca4b91c9-9a0e-4890-838d-b439ae6107e6

Zavattieri, L., D’Anna, S., \& Maillet, J. (2007). Evaluation of High-School Health Science Careers Program Impact on Student Retention and Careers. Journal of Allied Health, 36(2), 81-87.

\section{Appendix}

\section{Subset A}

Which of the following health care professionals would be responsible for administering lactated ringers to a patient?

- Nurse

- Medical Laboratory Scientist

- Occupational Therapist

- Respiratory Therapist

- Radiologic Technician

Anna Sikes is admitted to the hospital with a severe burn. Which of the following health care professionals would perform debridement and skin grafting?

- EMT's and Paramedics

- Medical Laboratory Scientist

- Nurses

- Radiologic Technologists

- $\quad$ Surgeons and Plastic Surgeons

Which health care professional would perform intubation on a burn patient and start mechanical ventilation?

- Medical Laboratory Scientist

- Nurse

- Occupational Therapist

- Radiology Technologist 
- Respiratory Therapist

A football player is injured on the field. An athletic trainer would:

- Assess field injuries and provide first response treatment

- Interpret results of diagnostic imaging

- Perform graft surgery to repair torn ACL

- Perform MRI studies

- Immediately remove the helmet and other protective gear to make the athlete as comfortable as possible

An athlete is injured on the field and requires an MRI scan of the knee. Which of the following health care professionals would perform the scan?

- Athletic trainer

- Nurse

- Physical Therapist

- Radiologic technologist

- Surgical technologist

Trevor Black has been admitted to the hospital with a high fever, rash, and cervical pain. The physician orders a total protein and gram stain on the spinal fluid. Which health care professional is responsible for testing the spinal fluid?

- Emergency room nurse

- Epidemiologist

- Medical Laboratory Scientist

- Neurologist

- Radiology technologist

Alfred was brought to the emergency room with pain in his jaw and right arm. Susie, another emergency room patient, has a fever and a sore throat. Which health care professional is responsible for prioritizing these patients as to the urgency of care?

- Cardiologist

- Emergency or critical care nurse

- Medical Laboratory Technologist

- Radiology technologist

- Respiratory therapist

John Smith has been experiencing fatigue, nausea, and diarrhea. His physician notes that he is severely jaundiced and suspects Hepatitis A. A Medical Laboratory Scientist would aid in the diagnosis of Hepatitis A by:

- Administering medications as necessary to relieve discomfort.

- Administering intravenous fluids.

- Performing immunologic marker studies and liver enzyme tests.

- Promoting sanitation in the community.

- Tracking down sources of infection during outbreaks.

Which of the following is NOT a responsibility of a Medical Records Technologist?

- Assign codes for diagnoses and procedures.

- Ensure medical records are complete.

- Make sure all forms are signed.

- Record vital signs such as recent blood pressure readings on patient's record.

- Use computer programs to tabulate and analyze data available on medical records.

Which health care professional would examine a biopsy of testicular tissue to determine the presence and type of testicular cancer? 
- Family physician

- Pathologist

- Radiologic technologist

- Surgeon

- Surgical technologist

Which health care professional would monitor a burn patient's resting metabolic rate and adjust nutrients in enteral tube feedings as needed?

- Dietician

- Nurse

- Occupational Therapist

- Physician

- Public Health professional

There has been an outbreak of Hepatitis A in the Salt Lake area. Which health professional would be responsible for identifying the source of the hepatitis and notifying individuals at risk of contracting the disease?

- Dietician

- Nurse

- Occupational Therapist

- Physician

- Public Health Professional

Which of the following health care personnel would be important in diagnosing and treating a patient with a recent myocardial infarction?

- Cardiologist

- Dietician

- Medical Laboratory Scientist

- Nurse

- Paramedic

- $\quad$ All of the above

\section{Subset B}

How much knowledge do you have of the responsibilities of a Medical Laboratory Scientist as part of the healthcare team?

- No knowledge

- I have heard of this profession

- I have some knowledge

- I know quite a bit about this profession

- I know a lot about this profession

How much knowledge do you have of the responsibilities of a Respiratory Therapist as part of the healthcare team?

- No knowledge

- I have heard of this profession

- I have some knowledge

- I know quite a bit about this profession

- I know a lot about this profession

How much knowledge do you have of the responsibilities of a Dietician as part of the healthcare team? 
- No knowledge

- I have heard of this profession

- I have some knowledge

- I know quite a bit about this profession

- I know a lot about this profession

How much knowledge do you have of the responsibilities of a Nurse as part of the healthcare team?

- No knowledge

- I have heard of this profession

- I have some knowledge

- I know quite a bit about this profession

- I know a lot about this profession

How much knowledge do you have of the responsibilities of a Physician as part of the healthcare team?

- No knowledge

- I have heard of this profession

- I have some knowledge

- I know quite a bit about this profession

- I know a lot about this profession

How much knowledge do you have of the responsibilities of a Radiology Technologist as part of the healthcare team?

- No knowledge

- I have heard of this profession

- I have some knowledge

- I know quite a bit about this profession

- I know a lot about this profession

How much knowledge do you have of the responsibilities of a Paramedic as part of the healthcare team?

- No knowledge

- I have heard of this profession

- I have some knowledge

- I know quite a bit about this profession

- I know a lot about this profession

How much knowledge do you have of the responsibilities of a Health Care Coder as part of the healthcare team?

- No knowledge

- I have heard of this profession

- I have some knowledge

- I know quite a bit about this profession

- I know a lot about this profession

How much knowledge do you have of the responsibilities of public health professionals as part of the healthcare team?

- No knowledge

- I have heard of this profession

- I have some knowledge

- I know quite a bit about this profession

- I know a lot about this profession

How much knowledge do you have of the responsibilities of a health care administrator as part of the healthcare team? 
- No knowledge

- I have heard of this profession

- I have some knowledge

- I know quite a bit about this profession

- I know a lot about this profession

\section{Subset C}

How likely are you to pursue a health profession following high school?

- Not likely

- Unsure but probably not

- I'm considering this

- Probably

- Definitely

\section{Copyrights}

Copyright for this article is retained by the author(s), with first publication rights granted to the journal.

This is an open-access article distributed under the terms and conditions of the Creative Commons Attribution license which permits unrestricted use, distribution, and reproduction in any medium, provided the original work is properly cited. 